\title{
The association of pregnancy-induced hypertension and neonatal hypospadias.
}

\author{
Yi Wang ${ }^{1}$, Lin Wang ${ }^{2}$, Zeyong Yang ${ }^{1}$, Fang Chen ${ }^{2}$, and Zhiwei Liu ${ }^{1}$ \\ ${ }^{1}$ International Peace Maternity and Child Health Hospital \\ ${ }^{2}$ Shanghai Sixth Peoples Hospital
}

February 5, 2021

\begin{abstract}
Background: The prevalence of hypertensive disorder in pregnancy has been well-documented worldwide. In Chinese newborns, the risk of hypospadias in women with hypertension during pregnancy remains ambiguous. This study aimed to evaluate the relationship between hypertension in pregnancy and neonatal hypospadias based on a large sample of Chinese people. Methods: A retrospective cohort study was conducted at our hospital from 2015 to 2019. Mothers who delivered male infants with hypospadias or those without any malformations were enrolled. Factors such as hypertension, placenta previa, thyroid diseases, hepatitis B, obesity, multiple birth, amniotic fluid, gestational age, birth weight, and in vitro fertilization were collected to establish a regression analysis to assess risk factors for hypospadias. Results: In total, 41,490 mothers and 42,244 male infants were enrolled. The overall incidence of hypospadias was $0.23 \%$. The occurrence rate of hypospadias in pregnancy-induced hypertension (PIH) group was higher than control group (0.944\% vs. $0.186 \%$, RR 5.08), whereas the occurrence rate in chronic hypertension group was $0 \%$. Potential exposure factors were screened for hypospadias, and PIH, multiple birth, hyperthyroidism, preterm delivery, low birth weight, and small for gestational age (SGA) were found to have higher proportion of hypospadias in offspring. After adjustment for potential confounders in the multivariate regression analysis, PIH (OR: 2.437, 95\% CI: $1.478-$ 4.016, P<0.01), birth weight (OR: 0.852, 95\% CI: 0.795-0.912, P<0.01), and SGA (OR: 3.282, 95\% CI: 1.644-6.549, P<0.01) showed a significant relationship with hypospadias. Conclusion: Women with PIH had higher risks of hypospadias in offspring. Lower birth weight, SGA and hyperthyroidism were also statistically associated with hypospadias.
\end{abstract}

\section{The association of pregnancy-induced hypertension and neonatal hypospadias}

Yi Wang1\# MD, Lin Wang 2,3,4\# MD, PhD, Zeyong Yang5, MD, PhD, Fang Chen 2,3,4* MD, PhD, Zhiwei Liu1* MD, PhD

1. Department of Neonatology, International Peace Maternity and Child Health Hospital, Shanghai Jiao Tong University School of Medicine, Shanghai, China. Email: wangyims@hotmail.com;

2. Department of Urology, Shanghai Sixth People's Hospital, Shanghai JiaoTong University, Shanghai, China. Email: wanglinbyg@163.com;

3. Department of Urology, Shanghai Children's Hospital, Shanghai Jiao Tong University, Shanghai, China; 4.Shanghai Eastern Institute of Urologic Reconstruction;

5. Department of Anesthesiology, International Peace Maternity and Child Health Hospital, Shanghai Jiao Tong University School of Medicine; Shanghai Key Laboratory of Embryo Original Disease; Shanghai Municipal Key Clinical Specialty, Shanghai, China. Email: zeyongyang@shsmu.edu.cn.

\# Yi Wang and Lin Wang contributed equally to this work.

\section{*Correspondence author:}


1.Zhiwei Liu MD, PhD, Department of Neonatology, International Peace Maternity and Child Health Hospital, Shanghai Jiao Tong University School of Medicine, Shanghai Key Laboratory of Embryo Original Disease, Shanghai Municipal Key Clinical Specialty, Huashan Rd. 1961 Shanghai, 200030, China; Tel: +86 21 64070434. Fax: +86 21 64474645. Email: liuzhiwei@hotmail.com.

2.Fang Chen, MD, PhD.Department of Urology, Shanghai Children's Hospital, Shanghai Jiao TongUniversity, 355 Luding Road, Shanghai, 200062, China; Department of Urology, Shanghai Sixth People's Hospital, Shanghai JiaoTong University, 600 Yishan Road, Shanghai, China;Shanghai Eastern Institute of Urologic Reconstruction.E-mail: doctorchenfang@126.com.

Funding : This work was supported by the National Key Research and Development Program of China (2016YFC1000203), the National Natural Science Foundation of China Grants (81974232), and the Shanghai Municipal Commission of Health and Family Planning (2016ZB0102-02).

Acknowledgements: The authors thank the participating parturients and their infants.

Ethics approval: Ethics approval and use of clinical data for research purposes was granted by the Ethics Committees of the International Peace Maternity and Child Health Hospital (GKLW 2017-101) in $23^{\text {rd }}$ August 2018.

Conflict of Interest: The authors declare that they have no competing interests.

\section{Abstract}

Background: The prevalence of hypertensive disorder in pregnancy has been well-documented worldwide. In Chinese newborns, the risk of hypospadias in women with hypertension during pregnancy remains ambiguous. This study aimed to evaluate the relationship between hypertension in pregnancy and neonatal hypospadias based on a large sample of Chinese people.

Methods: A retrospective cohort study was conducted at our hospital from 2015 to 2019. Mothers who delivered male infants with hypospadias or those without any malformations were enrolled. Factors such as hypertension, placenta previa, thyroid diseases, hepatitis B, obesity, multiple birth, amniotic fluid, gestational age, birth weight, and in vitro fertilization were collected to establish a regression analysis to assess risk factors for hypospadias.

Results: In total, 41,490 mothers and 42,244 male infants were enrolled. The overall incidence of hypospadias was $0.23 \%$. The occurrence rate of hypospadias in pregnancy-induced hypertension (PIH) group was higher than control group ( $0.944 \%$ vs. $0.186 \%$, RR 5.08), whereas the occurrence rate in chronic hypertension group was $0 \%$. Potential exposure factors were screened for hypospadias, and PIH, multiple birth, hyperthyroidism, preterm delivery, low birth weight, and small for gestational age (SGA) were found to have higher proportion of hypospadias in offspring. After adjustment for potential confounders in themultivariate regression analysis, PIH (OR: 2.437, 95\%CI: 1.478-4.016, P<0.01), birth weight (OR: 0.852, 95\%CI: 0.795$0.912, \mathrm{P}<0.01$ ), and SGA (OR: 3.282, 95\%CI: 1.644-6.549, $\mathrm{P}<0.01$ ) showeda significant relationship with hypospadias.

Conclusion: Women with PIH had higher risks of hypospadias in offspring. Lower birth weight, SGA and hyperthyroidism were also statistically associated with hypospadias.

\section{What's already known about this topic?}

Previous researches showed gestational hypertension was related with hypospadias in the offspring among Caucasian populations.

Placental dysfunction and abnormal placental blood flow perfusion, which were usually associated with PIH, might be the potential mechanisms for genital malformation.

What does this article add? 
Our findings suggested that PIH of the mother was an important independent risk factor for hypospadias in Chinese newborns, while chronic hypertension was irrelevant.

In addition, lower birth weight and SGA, which represent restricted growth of the fetus, and hyperthyroidism of the mother, were also significantly associated with hypospadias.

\section{Background}

Hypospadias is the most common type of male external genital malformation, and it typically manifests as simultaneous defects of the urethra and corpus spongiosum [4]. Patients with hypospadias are usually unable to urinate in a normal posture, and their sexual function may be affected in adulthood, which is not only inconvenient but also triggers anxiety and low self-esteem and may therefore cause serious psychological injury to patients and their families. Little is known about how to prevent or treat hypospadias due to the current lack of information regarding its mechanism and risk factors.

The incidence of hypospadias ranges from $0.3 \%-0.5 \%$, and this condition has recently shown an increasing trend $[1,2]$. Corrective surgeries have been attempted to improve the patients' appearance and function, but postoperative complicationscannot be completelyprevented [3]. Therefore, hypospadias is a serious social problem that impactsboth genital formation and the patients' psychology.

Previous research among Caucasian populations has showna relationship between gestational hypertension and hypospadias in offspring. It is known that placental dysfunction and abnormal placental blood flow perfusion caused by pregnancy-induced hypertension (PIH) are potential mechanisms for genital malformations $[6,7]$ and therefore may also contribute to the pathogenesis of hypospadias. Only limited data exist on the special risks and teratogenic effects of hypertension in pregnancy, and large studiesina Chinese population are extremely scarce. Therefore, the purpose of this study was to examine the relationship between hypospadias in Chinese newborns and hypertensive disorder during pregnancy to provide a perspective on the domestic clinical prevention of hypospadias.

\section{Materials and methods}

\section{Study design and population}

A retrospective cohort study was conducted on pregnancies and the corresponding incidence of genital malformationsin male neonates atthe International Peace Maternal and Child Health Hospital (affiliated withthe Shanghai Jiao Tong University School of Medicine) from January 2015 to December 2019 to observe the effect of hypertensive disorder in pregnancy on the development of neonatal hypospadias. Every step was carried out in accordance with the relevant guidelines. Ethics approval and use of clinical data for research purposes was granted by the Ethics Committees of the International Peace Maternity and Child Health Hospital. Patients with pregnancies who registered and obtained routine prenatal examinationsat our hospital and their male infants were enrolled in this study, including male infants with hypospadias diagnosed by neonatologists using clinical observations documented at birth. The analysis excluded pregnancies whose newborns had malformations other than hypospadias; these non-malformation infants and their mothers were used as the normal controls. Basedon the timing of the occurrence of hypertension, the pregnancies were divided into three groups: chronic hypertension ( $\mathrm{CH}$, hypertension appeared before 20 weeks gestation), pregnancy-induced hypertension (PIH, including gestational hypertension, pre-eclampsia, and eclampsia) and normal control groups (women without hypertension, proteinuria, or evidence of edema). For the risk factor analysis, the pregnancies were divided into a hypospadias group and a non-malformation group. This clinical study has been registered at .

\section{Exposure factors}

We collected the following information from our participants: maternal age, $\mathrm{CH}, \mathrm{PIH}$, placenta previa, multiple births, diabetes mellitus (DM), hyperthyroidism, hypothyroidism, hepatitis B, obesity, gestational age, amniotic fluid, newborn birth weight, birth weight for gestational age, and any use of assisted fertility techniques to estimate the relationship between maternal and fetal risk factors and hypospadias. The 
medical conditions mentioned above were obtained from the hospital database records, which were entered by obstetricians and neonatologists individually during the prenatal examinations and administration of the pregnancy. The diagnostic description was confirmed by the clinical findings, laboratory results, imaging, and pathological examinationsboth during pregnancy and after delivery according to the respective clinical protocols and guidelines.

\section{Statistical analysis}

We listed the number of infants with hypospadias in the $\mathrm{CH}$ group, PIH group, and control group to estimate the prevalence of hypospadias among pregnancies with or without hypertension. A t-test and singlefactor chi-square analysis were used for continuous variables and categorical data,respectively, to screen out potential exposure factors for hypospadias. These exposure factors, which showed significant differences upon single-factor analysis, were included in the follow-up binary logistic regression analysis for categorical variables and the linear regression for continuous variables. The crude regression analysis was conducted to estimate the prevalence of odds ratios (ORs) and 95\% confidence intervals (CI) for the associations of hypospadias with the different risk factors in the single-factor analysis. Multivariate regression analysis assessed the independent contribution of these factors to the risk of hypospadias. The Statistical Package for the Social Sciences software (version 25.0) was used to sort out and statistically analyze the relevant data. A significance level of $\mathrm{P}<0.05$ was set for all the tests.

\section{Results}

A total of 42,075 pregnancies produced 42,832 male infants from January 2015 to December 2019. There were588 male infants (including three pairs of twins) who had any other deformities except hypospadias, and we excluded these male infants and their mothers $(\mathrm{n}=585)$. Finally, 41,490pregnanciesand 42,244 male infants were enrolled (Figure 1). The prevalence of gestational hypertension and hypospadiasis shown in Table 1. In all, 97 newborns were diagnosed with hypospadias, and the overall incidence of hypospadias was $0.23 \%$ $(97 / 42,244)$. Of all the pregnant women, $119(0.29 \%)$ had $\mathrm{CH}, 2,648(6.38 \%)$ had PIH, and 38,723(93.33\%) had no evidence of hypertensionand were therefore used as the control. The occurrencerate of hypospadias was higher in the PIH group than in the control group (0.944\% vs. $0.186 \%$, respectively; RR: 5.08$)$, whereas the occurrence rate in the chronical hypertension group was $0 \%$ (Table 1 ).

The results of the t-test and chi-square test revealed that patients with PIH (25.8\% vs. $6.8 \%)$, multiple births ( $17.5 \%$ vs. $5.5 \%)$, hyperthyroidism ( $4.1 \%$ vs. $0.6 \%)$, preterm deliveries ( $30.9 \%$ vs. $9.0 \%)$, low-birth-weight (LBW) infants (34.0\% vs. $5.1 \%$ ), and SGA infants (27.8\% vs. $2.0 \%$ )hada higher incidence of hypospadias than the control participants $(\mathrm{P}<0.01$; Table 2$)$. The influence of the mothers' age, $\mathrm{CH}$, placenta previa, DM, hypothyroidism, hepatitis B, obesity, amniotic fluid, and use of in vitro fertilization (IVF)on the prevalence of hypospadias was not significant between groups $(\mathrm{P}>0.05)$.

To estimate the contribution of these factors on hypospadias, a binary logistic regression analysis was conducted for categorical variables (PIH, singleton births, hyperthyroidism, SGA), and a linear regression was also carried out for continuous variables (gestational age, birth weight; Table3). These factors still showed a significant relationship with hypospadias in this crude model. However, after adjustment for these potential confounders in a multivariate logistic regression analysis, the results indicated that singleton births (OR: $1.215,95 \%$ CI: $0.672-2.196, \mathrm{P}=0.520$ ) and gestational age (OR: 1.138, 95\% CI: 0.982-1.319, $\mathrm{P}=0.087$ ) failed to maintain statistical significance, whereas PIH (OR: 2.437, 95\% CI: 1.478-4.016, P<0.01), hyperthyroidism (OR: 4.008, 95\% CI: 1.364-11.773, P=0.012), LBW (OR: 0.852, 95\%CI: 0.795-0.912, $\mathrm{P}<0.01$ ), and SGA (OR: 3.282, 95\%CI: 1.644-6.549, $\mathrm{P}<0.01$ ) wereindependent factors that contributed to hypospadias.

\section{Discussion}

Prior studies have suggesteda certain relationship between hypertensive disorders in pregnancy and the occurrence of hypospadias in Caucasian populations [11, 12]. However, there is still a lack of large-scale clinical research related to this condition in China. Our study was based on a retrospective cohort study of 41,490 maternal patients and their 42,244newborns, and it found that the occurrence rate of hypospadias 
also had a significant correlation with PIH inChina. In this study, PIH caused a significant increase in the incidence of hypospadias in male newborns that reached $0.944 \%$, which was about five times as highas the rate in the normotensive pregnancies in the control group (0.186\%). Moreover, the statistical analysis showed that the risk of hypospadias caused by PIH was still more than three times as high after multi-factor correction.Interestingly, no newborn with hypospadias was identified in the group with $\mathrm{CH}$ in this study, which may have been influenced by the difference in the etiology of these two conditions. $\mathrm{CH}$ and PIH share common symptoms of high blood pressure, butthe former is a type of vascular disease that is mostly related with atherosclerosis, psychological factors, or genetic factors, while PIH is typically associated with gestational status and may be caused by placental dysfunction. This study may help explain the potential pathogenesis of hypospadias related with HIP.

The key time window for male reproductive organ development is from 8-14 weeks of gestation during early pregnancy. There is evidence [8] that the morphogenesis of the male reproductive organs depends on a series of hormonal stimulations in which placental-derived hormones play a key role. At first, the human chorionic gonadotropin hormone secreted by the placenta in the early embryo stimulates the differentiation of testicular stromal cells. By the eighth week of pregnancy, testicular stromal cells begin secreting testosterone, which is essential for the normal development of the penis and testes. Therefore, placental dysfunction in early pregnancy could be a possible pathogenic factor for hypospadias. Placental dysfunction was common in patients with PIH [9], which indicated that PIH was relevant to the pathogenesis of hypospadias. In many pregnancies with PIH, especially preeclampsia, early placental function is seriously affected. Hypertension caused by placental spiral, insufficient arterial invasion, luminal contraction and stenosis, or inadequate placental blood flow perfusion may lead to important changes in placental function and consequently affect fetal growth and development $[9,10]$. Although PIH is usually diagnosed after 20 weeksof gestation (after the key time window of hypospadias development), the condition may beginlong before 20 weeks, which means that PIH may impact urologic development substantially earlier than it is diagnosed. It is still unknown whether hypospadias occurs secondary to PIH or whether both are outcomes of a common pathogeny. Further genetic or pathologic studies should reveal the potential pathogenesis.

The proportions of hypospadias in LBW and SGA neonates were significantly higher than those in normalweight neonates. LBW is usuallyrelated with premature delivery, which is a common complication in patients who have hypertensive disorders in pregnancy. A multivariate regression analysis indicated thatgestational age showed no significant relationship with hypospadias after adjustment for confounding factors. However, a decreasing birth weight still increased the incidence of hypospadias in the multivariate regression analysis. In addition, SGA was determined to be an independent risk factor for hypospadias regardless of gestational age. This finding suggested that chronic intrauterine distress might predict the risk of hypospadias in the male fetus despite the gestational age. The birth weight for gestational age could also reflect the condition of fetal growth in the uterus at every stage of gestation; the most intuitive manifestation of fetal growth restriction is that the fetal weight, body length, and biparietal diameter during pregnancy arelower than the normal average [15]. The rate of hypospadias after birth was as high as $1.9 \%$ in the group with limited fetal growth during pregnancy[15]. Therefore, fetal weight assessment, body length, and biparietal diameter monitoring during pregnancy can play a vital role in predicting the risk of hypospadias. Our findingsfrom this study may contribute to further information about this condition and a better understanding of how it develops.

The presence ofhyperthyroidism during pregnancy also showed a significantlyincreased risk of hypospadias in newborns in our study. The relationship between neonatal malformations and hyperthyroidism that is treated during pregnancy is still controversial. Most scholars believe that the drugs used to treat hyperthyroidism are more likely to be teratogenic; for example, propylthiouracil is recommended to treat hyperthyroidism during pregnancy [13, 14]. Additionally, the timing of the onset of hyperthyroidism and beginning its associatedmedication may also affect the pathogenesis of hypospadias. Our study showed that the $95 \%$ CIsspanned a wide range from 1.364-11.773, which may have decreased the confidence level. Studies with further classification of thyroid diseases and the medications that pregnant women take for themcouldbetter explain their relationship with hypospadias with more certainty. 
IVF did not increase the risk of hypospadias in newborns, and this result is consistent with those of previous studies [16]. The risk of hypospadias in the offspring of older women was significantly increased according to the statistics of California Birth Defects Monitoring[17]. However, this present study did not reach a similar conclusion. The statistical results showed that there was no significant difference in maternal age between the two groups.

One limitation of our study was that we did include any information about family history, hormonal medications, exposure to toxic and hazardous substances, or the history of previous abortions. During the process of data collection, we also found that the information we already had might not be correct due to a consideration for the patients' personal privacy or inadequate medical history collecting. Therefore, to avoid inaccuracies, we did not include these data. Additionally, the classification of hypertensive disorder in pregnancy might need more refinement. Further work may require optimized classifications and a more comprehensive design to provide deep insight into the complex etiology of hypospadias.

\section{Conclusion}

Our findings suggested that maternal PIH was an important independent risk factor for hypospadias in Chinese newborns, while CH showed no relationship with hypospadias in the offspring of mothers with PIH. In addition, LBW and SGA (which indicaterestricted growth of the fetus) and hyperthyroidism of the mother werealso significantly associated with hypospadias.

\section{References:}

[1] Baskin L S, Ebbers M B. Hypospadias: anatomy, etiology, and technique. Journal of pediatric surgery, 2006, 41(3): 463-72.

[2] Sørensen H T, Pedersen L, Nørgaard M, et al. Maternal asthma, preeclampsia and risk of hypospadias. Epidemiology, 2005, 16(6): 806-7.

[3] Lucas J, Hightower T, Weiss D A, et al. Time to Complication Detection after Primary Pediatric Hypospadias Repair: A Large, Single-Center, Retrospective Cohort Analysis. J Urol, 2020, 101097ju0000000000000762.

[4] Brouwers M M, Feitz W F, Roelofs L A, et al. Risk factors for hypospadias. Eur J Pediatr, 2007, 166(7): 671-8.

[5] Roberts C L, Ford J B, Algert C S, et al. Population-based trends in pregnancy hypertension and preeclampsia: an international comparative study. BMJ Open, 2011, 1(1): e000101.

[6] Yinon Y, Kingdom J C, Proctor L K, et al. Hypospadias in males with intrauterine growth restriction due to placental insufficiency: the placental role in the embryogenesis of male external genitalia. Am J Med Genet A, 2010, 152a(1): 75-83.

[7] Van Zutphen A R, Werler M M, Browne M M, et al. Maternal hypertension, medication use, and hypospadias in the National Birth Defects Prevention Study. Obstet Gynecol, 2014, 123(2 Pt 1): 309-17.

[8] Arendt L H, Henriksen T B, Lindhard M S, et al. Hypertensive Disorders of Pregnancy and Genital Anomalies in Boys: A Danish Nationwide Cohort Study . Epidemiology, 2018, 29(5): 739-48.

[9] Culver J C, Dickinson M E. The effects of hemodynamic force on embryonic development. Microcirculation, 2010, 17(3): 164-78.

[10] Mayhew T M, Charnock-Jones D S, Kaufmann P. Aspects of human fetoplacental vasculogenesis and angiogenesis. III. Changes in complicated pregnancies. Placenta, 2004, 25(2-3): 127-39.

[11] Agopian A J, Hoang T T, Mitchell L E, et al. Maternal hypertension and risk for hypospadias in offspring. Am J Med Genet A, 2016, 170(12): 3125-32. 
[12] Sheriff F R, Lopez A, Lupo P J, et al. Maternal hypertension and hypospadias in offspring: A systematic review and meta-analysis. Birth Defects Res, 2019, 111(1): 9-15.

[13] Andersen S L, Olsen J, Wu C S, et al. Severity of birth defects after propylthiouracil exposure in early pregnancy. Thyroid, 2014, 24(10): 1533-40.

[14] Laurberg P, Andersen S L. Therapy of endocrine disease: antithyroid drug use in early pregnancy and birth defects: time windows of relative safety and high risk?. Eur J Endocrinol, 2014, 171(1): R13-20.

[15] Hussain N, Chaghtai A, Herndon C D, et al. Hypospadias and early gestation growth restriction in infants. Pediatrics, 2002, 109(3): 473-8.

[16] Massaro P A, MacLellan D L, Anderson P A, et al. Does intracytoplasmic sperm injection pose an increased risk of genitourinary congenital malformations in offspring compared to in vitro fertilization? A systematic review and meta-analysis. J Urol, 2015, 193(5 Suppl): 1837-42.

[17] Fisch H, Golden R J, Libersen G L, et al. Maternal age as a risk factor for hypospadias. The Journal of urology, 2001, 165(3): 934-6.

\section{Hosted file}

flow chart with legend.pdf available at https://authorea.com/users/393863/articles/507372the-association-of-pregnancy-induced-hypertension-and-neonatal-hypospadias

\section{Hosted file}

Table 1.pdf available at https://authorea.com/users/393863/articles/507372-the-associationof-pregnancy-induced-hypertension-and-neonatal-hypospadias

\section{Hosted file}

Table 2.pdf available at https://authorea.com/users/393863/articles/507372-the-associationof-pregnancy-induced-hypertension-and-neonatal-hypospadias

\section{Hosted file}

Table 3.pdf available at https://authorea.com/users/393863/articles/507372-the-associationof-pregnancy-induced-hypertension-and-neonatal-hypospadias 